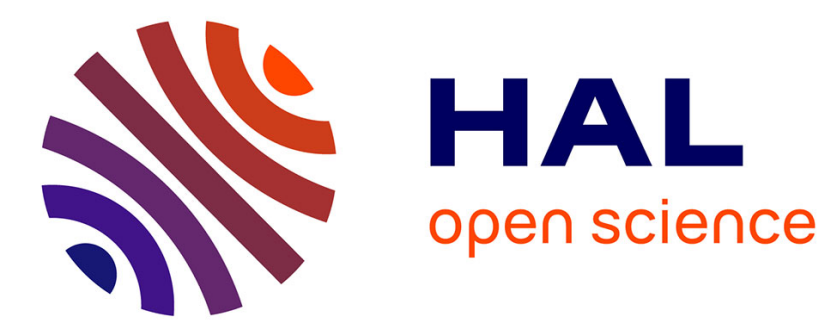

\title{
A Formalism for Stochastic Adaptive Systems
}

\author{
Benoît Boyer, Axel Legay, Louis-Marie Traonouez
}

\section{To cite this version:}

Benoît Boyer, Axel Legay, Louis-Marie Traonouez. A Formalism for Stochastic Adaptive Systems. Leveraging Applications of Formal Methods, Verification and Validation. Specialized Techniques and Applications, Oct 2014, Corfu, Greece. pp.160 - 176, 10.1007/978-3-662-45231-8_12 . hal-01087327

\section{HAL Id: hal-01087327 https://hal.science/hal-01087327}

Submitted on 25 Nov 2014

HAL is a multi-disciplinary open access archive for the deposit and dissemination of scientific research documents, whether they are published or not. The documents may come from teaching and research institutions in France or abroad, or from public or private research centers.
L'archive ouverte pluridisciplinaire HAL, est destinée au dépôt et à la diffusion de documents scientifiques de niveau recherche, publiés ou non, émanant des établissements d'enseignement et de recherche français ou étrangers, des laboratoires publics ou privés. 


\title{
A Formalism for Stochastic Adaptive Systems
}

\author{
Benoît Boyer, Axel Legay, and Louis-Marie Traonouez \\ Inria / IRISA, Campus de Beaulieu, 35042 Rennes CEDEX, France
}

\begin{abstract}
Complex systems such as systems of systems result from the combination of several components that are organized in a hierarchical manner. One of the main characteristics of those systems is their ability to adapt to new situations by modifying their architecture. Those systems have recently been the subject of a series of works in the software engineering community. Most of those works do not consider quantitative features. The objective of this paper is to propose a modeling language for adaptive systems whose behaviors depend on stochastic features. Our language relies on an extension of stochastic transition systems equipped with (1) an adaptive operator that allows to reason about the probability that a system has to adapt its architecture over time, and (2) dynamic interactions between processes. As a second contribution, we propose a contract-based extension of probabilistic linear temporal logic suited to reason about assumptions and guarantees of such systems. Our work has been implemented in the PLASMA-LAB tool developed at Inria. This tool allows us to define stochastic adaptive systems with an extension of the Prism language, and properties with patterns. In addition, Plasma-LAB offers a simulation-based model checking procedure to reason about finite executions of the system. First experiments on a large case study coming from an industrial driven European project give encouraging results.
\end{abstract}

\section{Context}

Critical systems increasingly rely on dynamically adaptive programs to respond to changes in their physical environments. Reasoning about such systems require to design new verification techniques and formalisms that take this model of reactivity into account [7].

This paper proposes a complete formalism for the rigorous design of stochastic adaptive systems (SAS), whose components' behaviors and environment changes are represented via stochastic information. Adding some stochastic feature to components' models is more realistic, especially regarding the environment aspect, e.g. the probability of hardware failure, the fire frequency in a forest or a growing city population...

We view the evolution of our system as a sequence of views, each of them representing a topology of the system at a given moment of time. In our setting, views are represented by a combination of Markov chains, and stochastic adaptive transitions that describe the environment evolution as transitions between different views of the SAS (e.g. adding or removing components). Each view thus associates the new environment behaviour and a new system configuration (a new 


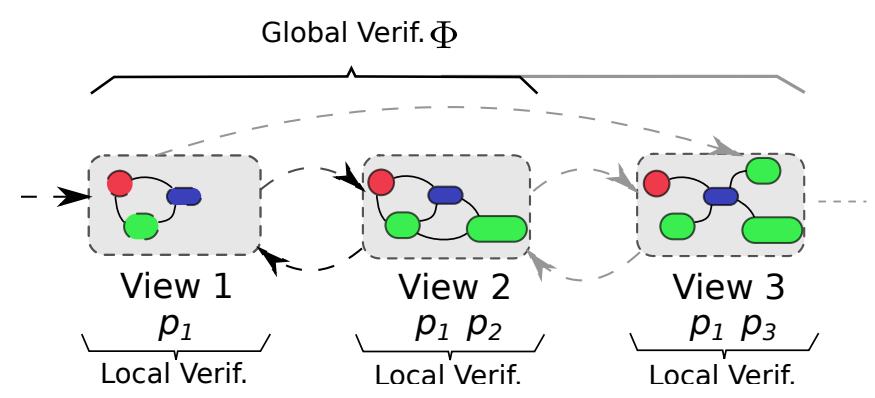

Fig. 1. Illustration of SAS Methodology

topology, addition or suppression of system components...). The incremental design is naturally offered to the system architect who can extend easily an existing model by creating new views.

Properties of views can be specified with Bounded Linear Temporal Logic (BLTL) that allows to reason about finite execution. To reason about sequences of view, we propose Adaptive BLTL (A-BLTL) that is an extension of BLTL with an adaptive operator to reason about the dynamic change of views. We also show that the formalism extend to contracts that permit to reason about both assumptions and guarantees of the system.

Consider the system described in Figure 1. This system is composed by three different views linked by adaptive transitions (represented by dashed arrows). Each view contains some system components in a particular topology denoting a configuration of the SAS. Each local property $p_{1}, p_{2}, p_{3}$ is attached to one or more views. There is also global property $\Phi$. The SAS is initially designed by View 1 and View 2 and the black dashed arrows. Properties $p_{1}, p_{2}$ are validated for the corresponding views and $\Phi$ is validated against this complete initial version of the system. To fit with the upcoming settings of the system, the system architect updates the model by adding View 3 with new adaptive transitions (in grey). This requires to only validate $p_{1}$ and $p_{3}$ against View 3 and to validate again the global property $\Phi$ against the system including all the three views.

We propose a new Statistical Model Checking (SMC) [22,20] algorithm to compute the probability for a SAS to satisfy an A-BLTL property. SMC can be seen as a trade-off between testing and formal verification. The core idea of SMC is to generate a number of simulations of the system and verify whether they satisfy a given property expressed in temporal logics, which can be done by using runtime verification approaches [12]. The results are then used together with algorithms from the statistical area in order to decide whether the system satisfies the property with some probability. One of the key points to implement an SMC algorithm is the ability to bound a priori the size of the simulation, which is an important issue. Indeed, although the SAS can only spend a finite amount of time in a given view, the time bound is usually unknown and simulation cannot be bounded. To overcome the problem, we expand on the work of Clarke [21] 
and consider a combination of SMC and model checking algorithm for untimed systems.

As a second contribution, we propose high-level formalisms to represent both SAS and A-BLTL/contracts. The formalism used to specify SAS relies on an extension of the Reactive Module Language (RML) used by the popular Prism toolset [16]. Properties are represented with an extension of the Goal and Contract Specification Language (GCSL) [2] defined in the DANSE IP project [11]. This language offers English-based pattern to reason about timed properties without having to learn complex mathematics inherent to any logic.

Finally, as a last contribution, we have implemented our work in PLASMA-LAB [5] - a new powerful SMC model checker. The implementation has been tested on a realistic case study defined with industry partners of DANSE.

\section{Modeling Stochastic Adaptive Systems}

In this section, we present the formal model used to encode behaviors of adaptive systems. In Section 2.1, we introduce Markov chains (MC) to represent individual components of a view. Then, in Section 2.2, we show how to describe views as well as relations between views, i.e., adaptive systems.

\subsection{Discrete and Continuous Time Markov Chains}

Definition 1. A (labelled) transition system is a tuple $\mathcal{T}=(Q, \bar{q}, \Sigma, \rightarrow, A P, L)$ where $Q$ is a set of states, $\bar{q} \in Q$ is the initial state, $\Sigma$ is a finite set of actions, $\rightarrow: Q \times \Sigma \times Q$ is the transition relation, $A P$ is a set of atomic propositions, and $L: Q \rightarrow 2^{A P}$ is a state labelling function that assigns to each state the set of propositions that are valid in the state.

We denote by $q \stackrel{a}{\longrightarrow} q^{\prime}$ the transition $\left(q, a, q^{\prime}\right) \in \rightarrow$. A trace is a finite or infinite alternating sequence of states and time stamps $\rho=t_{0} q_{0} t_{1} q_{1} t_{2} q_{2} \ldots$, such that $\forall i . \exists a_{i} \in \Sigma . q_{i} \stackrel{a_{i}}{\longrightarrow} q_{i+1}$. Time stamps measure the cumulative time elapsed from a time origin. In discrete time models delays are integer values (i.e., $t_{0}=0$, $t_{1}=1, t_{2}=2$ ) and therefore they can be omitted. In continuous time models they are real values. We denote by $|\rho|$ the length of a trace $\rho$. If $\rho$ is infinite then $|\rho|=\infty$. A trace is initial if $q_{0}=\bar{q}$ and $t_{0}=0$. We denote by $\operatorname{trace}_{n}(\mathcal{T})$ (resp. $\operatorname{trace}(\mathcal{T}))$ the set of all initial traces of length $n$ (resp. infinite traces) in $\mathcal{T}$. Let $0 \leq i \leq|\rho|$, we denote $\rho_{\mid i}=t_{0} q_{0} t_{1} q_{1} \ldots t_{i-1} q_{i-1}$ the finite prefix of $\rho$ of length $i, \rho^{\mid i}=t_{i} q_{i} t_{i+1} q_{i+1} \ldots$ the suffix of $\rho$ that starts at position $i$, and $\rho[i]=q_{i}$ the state at position $i$.

We now extend transition systems with probabilities to represent uncertainty of behaviors or of material on which the system is running. We present two semantics, either with discrete or continuous time, that are both compatible with our setting. A discrete time Markov chain (DTMC) is a state-transition system in which each transition is labelled by a probability $\mathbf{P}\left(s, s^{\prime}\right)$ to take the transition from state $s$ to state $s^{\prime}$. 
Definition 2. A (labelled) DTMC is a tuple $\mathcal{D}=(Q, \bar{q}, \Sigma, \rightarrow, A P, L, \mathbf{P})$ where:

- $(Q, \bar{q}, \Sigma, \rightarrow, A P, L)$ is a labelled transition system,

- $\mathbf{P}: Q \times Q \rightarrow[0,1]$ is a transition probability matrix, such that $\sum_{q^{\prime} \in Q} \mathbf{P}\left(q, q^{\prime}\right)=$ 1 for all $q \in Q$,

$-\rightarrow$ is such that $q \stackrel{a}{\longrightarrow} q^{\prime}$ iff $\mathbf{P}\left(q, q^{\prime}\right)>0$, and for each state $q$ there is at most one action $a \in \Sigma$ such that $q \stackrel{a}{\longrightarrow} q^{\prime}$ for some $q^{\prime}$.

In continuous time Markov chains (CTMCs) transitions are given a rate. The sum of rates of all enabled transitions specifies an exponential distribution that determines a real value for the time spent in the current state. The ratio of the rates then specifies which discrete transition is chosen.

Definition 3. A (labelled) $C T M C$ is a tuple $\mathcal{C}=(Q, \bar{q}, \Sigma, \rightarrow, A P, L, \mathbf{R})$ where:

- $(Q, \bar{q}, \Sigma, \rightarrow, A P, L)$ is a labelled transition system,

- $\mathbf{R}: Q \times Q \rightarrow \mathbb{R}_{\geq 0}$ is a transition rate matrix,

$-\rightarrow$ is such that $q \stackrel{a}{\longrightarrow} q^{\prime}$ iff $\mathbf{R}\left(q, q^{\prime}\right)>0$, and there is a unique $a \in \Sigma$ such that $q \stackrel{a}{\longrightarrow} q^{\prime}$.

In our setting, a view of a system is represented by the combination of several components. We can compute the parallel composition $C_{1} \| C_{2}$ of two DTMCs (resp. CTMCs) defined over the same alphabet $\Sigma$. Let $\left(Q_{1}, \bar{q}_{1}, \Sigma, \rightarrow_{1}\right.$ , $\left.A P_{1}, L_{1}\right)$ and $\left(Q_{2}, \bar{q}_{2}, \Sigma, \rightarrow_{2}, A P_{2}, L_{2}\right)$ be the two underlying transition systems. We first compute their parallel composition, which is a labelled transition system $(Q, \bar{q}, \Sigma, \rightarrow, A P, L)$, where $Q=Q_{1} \times Q_{2}, \bar{q}=\left(\bar{q}_{1}, \bar{q}_{2}\right), A P=A P_{1} \cup A P_{2}, L(q)=$ $L_{1}\left(q_{1}\right) \cup L_{2}\left(q_{2}\right)$ and the transition relation $\rightarrow$ is defined according to the following rule:

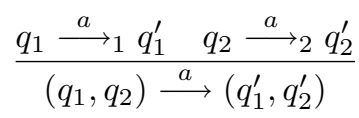

Then, in case of DTMCs, the new transition probability matrix is such that $\mathbf{P}\left(\left(q_{1}, q_{2}\right),\left(q_{1}^{\prime}, q_{2}^{\prime}\right)\right)=\mathbf{P}\left(q_{1}, q_{1}^{\prime}\right) * \mathbf{P}\left(q_{2}, q_{2}^{\prime}\right)$, and in case of CTMCs the new transition rate matrix is such that $\mathbf{R}\left(\left(q_{1}, q_{2}\right),\left(q_{1}^{\prime}, q_{2}^{\prime}\right)\right)=\mathbf{R}\left(q_{1}, q_{1}^{\prime}\right) * \mathbf{R}\left(q_{2}, q_{2}^{\prime}\right)$. DTMCs with different alphabets can also be composed and they synchronize on common actions. However, if both DTMCs can perform a non synchronized action, a uniform distribution is applied to resolve the non determinism. In case of CTMCs, the two actions are in concurrence, such that if $q_{1} \stackrel{a}{\longrightarrow} 1 q_{1}^{\prime}$ with $a \notin \Sigma_{2}$, then $\left(q_{1}, q_{2}\right) \stackrel{a}{\longrightarrow}\left(q_{1}^{\prime}, q_{2}\right)$ and $\mathbf{R}\left(\left(q_{1}, q_{2}\right),\left(q_{1}^{\prime}, q_{2}\right)\right)=\mathbf{R}\left(q_{1}, q_{1}^{\prime}\right)$. In what follows, we denote by Sys $=C_{1}\left\|C_{2}\right\| \cdots \| C_{n}$ the DTMC (resp. CTMC) that results from the composition of the components $C_{1}, C_{2}, \ldots, C_{n}$.

\subsection{Stochastic Adaptive Systems (SAS)}

An adaptive system consists in several successive views. It starts in an initial view that evolves until it reaches a state in which an adaptation is possible. This adaptation consists in a view change that depends on a probability distribution that represents uncertainty of the environment. 
Definition 4. A $S A S$ is a tuple $(\Delta, \Gamma, S, \overline{\text { sys }}, \rightsquigarrow)$ where:

- $\Delta=\left\{C_{1}, C_{2}, \ldots, C_{n}\right\}$ is a set of DTMCs (resp. CTMCs) that are the components of the $S A S$.

- $\Gamma$ is the set of views of the $S A S$, such that each view is a stochastic system obtained from the parallel composition some components from $\Delta$.

$-\overline{s y s} \in \Gamma$ is the initial view.

- $S$ is the set of states of the $S A S . S$ is the union of the states of each view in $\Gamma$, i.e., for each state $s \in S$ there exists $\left\{C_{1}, C_{2}, \ldots, C_{k}\right\} \subseteq \Delta$ such that $s \in Q_{1} \times Q_{2} \times \cdots \times Q_{k}$ (where $\forall i, 1 \leq i \leq k, Q_{i}$ is the set of states of $C_{i}$ ).

$-\rightsquigarrow \subseteq S \times[0,1]^{S}$ is a set of adaptive transitions.

Observe that the number of components per state may vary. This is due to the fact that different views may have different components. Observe also that it is easy to add new views to an existing adaptive system without having to re-specify the entire set of views. An element $(s, \mathbf{p}) \in \rightsquigarrow$ consists in a state $s$ from a view sys $\in \Gamma$ and a probability distribution $\mathbf{p}$ over the states in $S$. When $s \neq s^{\prime}$, we denote $s \rightsquigarrow s^{\prime}$ if there exists $\mathbf{p}$ such that $(s, \mathbf{p}) \in \rightsquigarrow$ and $\mathbf{p}\left(s^{\prime}\right)>0$, which means that state $s$ can be adapted into state $s^{\prime}$ with probability $\mathbf{p}\left(s^{\prime}\right)$.

A trace $\rho$ in a SAS is either a finite combination of $n$ traces $\rho=\rho_{0} \rho_{1} \ldots \rho_{n}$, such that for all $0 \leq i \leq n-1, \rho_{i}=t_{0 i} s_{0 i} t_{1 i} s_{1 i} \ldots t_{l i} s_{l i}$ is a finite trace of sys $_{i} \in \Gamma$, and $s_{l i} \rightsquigarrow s_{0 i+1}$, and $t_{0 i+1}=0$, and $\rho_{n}$ is a finite or infinite trace sys $_{n} \in \Gamma$. Otherwise $\rho$ may be an infinite combination of finite traces $\rho=\rho_{0} \rho_{1} \ldots$ that satisfy for all $i$ the same constraints.

\section{A logic for SAS properties}

\subsection{Probabilistic Adaptive Bounded Linear Temporal Logic}

We consider quantitative verification of dynamic properties that are expressed via a quantitative extension of the Adaptive Linear Temporal Logic (A-LTL) proposed in [23]. Our logic, which we call Adaptive Bounded Linear Temporal Logic (A-BLTL), relies on an extension of Bounded Linear Temporal Logic (BLTL) combined with an adaptive operator. Although the logic is not strictly more expressive than BLTL, it is more suitable to describe properties of individual views, as well as global properties of the adaptive system, and it allows to develop specific algorithms for these properties. In the last part of the section, we also show how one can define contracts on such logic, where a contract [17] is a pair of assumptions/guarantees that must be satisfied by the system.

We first introduce BLTL, a logic used to express properties on individual views. In BLTL, formulas are built by using the standard Boolean connectors $\wedge, \vee, \Longrightarrow, \neg$, and the temporal operators $G, F, X, U$ borrowed from Linear Temporal Logic (LTL). The main difference between BLTL and classical LTL is that each temporal modality is indexed by a bound $k$ that defines the length of the run on which the formula must hold. The semantics of a BLTL formula is defined in Table 1 for finite executions of CTMC/DTMC $\rho=t_{0} s_{0} t_{1} s_{1} t_{2} s_{2} \ldots$, 


\begin{tabular}{|ll|l|}
\hline$\rho \models X_{\leq k} \Phi$ & $\equiv \exists i, i=\max \left\{j \mid t_{0} \leq t_{j} \leq t_{0}+k\right\} \quad$ and $\rho^{\mid i} \models \Phi$ \\
$\rho \models \Phi_{1} U_{\leq k} \Phi_{2} \equiv \exists i, t_{0} \leq t_{i} \leq t_{0}+k \quad$ and $\quad \rho^{\mid i} \models \Phi_{2} \quad$ and $\forall j, 0 \leq j \leq i, \rho^{\mid j} \models \Phi_{1}$ \\
\hline$\rho \models \Phi_{1} \wedge \Phi_{2} \quad \equiv \rho \models \Phi_{1}$ and $\rho \models \Phi_{2}$ & $\rho \models \neg \Phi \quad \equiv \rho \not \models \Phi$ \\
$\rho \models P$ & $\equiv P \in L\left(s_{0}\right)$ & $\rho \models$ true $\quad \rho \not \models$ false \\
\hline
\end{tabular}

Table 1. Semantics of BLTL

with $|\rho| \geq k$. If $|\rho|<k$, it is extended by duplicating the last state enough times. In case formulas are nested, the value of $k$ adapts incrementally.

We now generalize BLTL to adaptive systems. For doing so, we introduce an adaptive operator in the spirit of [24]. The new logic A-BLTL is an extension of BLTL with an adaptive operator $\Phi \stackrel{\Omega}{\Rightarrow} \leq k \Psi$, where $\Phi$ is a BLTL formula, $\Psi$ is an A-BLTL formula, $\Omega$ is a predicate over the states of different views of the SAS, and $k$ is a time bound that limits the execution time of the adaptive transition. We will also consider unbounded versions of the adaptive operator.

Definition 5 (A-BLTL semantics). Let $\Phi \stackrel{\Omega}{\Rightarrow} \leq k \Psi$ be an $A$-BLTL formula and $\rho=t_{0} s_{0} t_{1} s_{1} t_{2} s_{2} \ldots$ be an execution of the $S \bar{A} S$ :

$$
\begin{aligned}
& \rho \models \Phi \stackrel{\Omega}{\Rightarrow} \leq k \Psi \equiv \exists i, i=\min \left\{j \mid t_{0} \leq t_{j-1} \leq t_{0}+k \wedge\right. \\
& \left.\rho_{\mid j} \models \Phi \wedge s_{j-1} \rightsquigarrow s_{j} \wedge \Omega\left(s_{j-1}, s_{j}\right)\right\} \wedge \rho^{\mid i} \models \Psi
\end{aligned}
$$

The property is unbounded if $k=\infty$, and in that case we write $\Phi \stackrel{\Omega}{\Rightarrow} \Psi$.

According to Definition 5 , an execution $\rho$ satisfies an adaptive formula $\Phi \stackrel{\Omega}{\Rightarrow} \leq k$ $\Psi$ if and only if there exists a minimal prefix of $\rho$ that satisfies $\Phi$ and reaches a state $s_{j-1}$, such that $\Omega\left(s_{j-1}, s_{j}\right)$ is satisfied, and such that the suffix of $\rho$ from state $s_{j}$ satisfies $\Psi$. Therefore to satisfy this formula it is necessary to observe an adaptation compatible with $\Omega$. We relax this constraint by introducing a new operator $\Phi \stackrel{\Omega}{\longrightarrow} \leq k \Psi$, for which an adaptation is not necessary but triggers a check of $\Psi$ when it happens. It is equivalent to the following formula: $\Phi \stackrel{\Omega}{\longrightarrow} \leq k \Psi \equiv$ $(\Phi \stackrel{\Omega}{\Rightarrow} \leq k$ true $) \Longrightarrow(\Phi \stackrel{\Omega}{\Rightarrow} \leq k \Psi)$.

We finally introduce stochastic contracts, that are used to reason about both the adaptive system and its environment via assumptions and guarantees.

Definition 6 (Contracts for SAS). A contract is defined as a pair $(A, G)$, where $A$ and $G$ are respectively called the Assumption and the Guarantee. $A$ SAS $\mathcal{M}$ satisfies the contract $(A, G)$ iff $\forall \rho, \rho \models A \Rightarrow \rho \models G$, where $\rho$ is a trace of $\mathcal{M}$ and $\rho \models A$ (resp. $G$ ) means the trace $\rho$ satisfies the assumption $A$ (resp. the guarantee $G)$. In that case we write $\mathcal{M} \models(A, G)$.

\subsection{Verifying SAS Properties using SMC}

SMC $[22,20]$ is an alternative to model checking [3,9] that employs Monte Carlo methods to avoid the state explosion problem. SMC estimates the probability that 
a system satisfies a property using a number of statistically independent simulation traces of an executable model. By using results from the statistic area, SMC decides whether the system satisfies the property with some degree of confidence, and therefore it avoids an exhaustive exploration of the state-space of the model that generally does not scale up. It has already been successfully experimented in biology area $[10,15,16]$, software engineering [8] as well as industrial area like aeronautics [4].

The basic algorithm used in SMC is the Monte Carlo algorithm. This algorithm estimates the probability that a system Sys satisfies a BLTL property $P$ by checking $P$ against a set of $N$ random executions of $S y S$. The estimation $\hat{p}$ is given by $\hat{p}=\frac{\sum_{1}^{N} f\left(\rho_{i}\right)}{N}$ where $f\left(\rho_{i}\right)=1$ if $\rho_{i} \models P, 0$ otherwise.

Using the formal semantics of BLTL, each execution trace $\rho_{i}$ is monitored in order to check if $P$ is satisfied or not. The accuracy of the estimation increases with the number of monitored simulations. This accuracy can be controlled thanks to the Chernoff-Hoeffding bound [14]. It relates $N$ to $\delta$ and $\varepsilon$, that are respectively the confidence and the error bound of $\hat{p}$ :

$$
\operatorname{Pr}(|p-\hat{p}|<\varepsilon) \geq 1-\delta \quad \text { if } \quad N \geq \frac{\ln \left(\frac{2}{\delta}\right)}{2 \varepsilon^{2}}
$$

According to this relation, the user is able to trade off analysis time in return for accuracy of the result using the parameters $\delta$ and $\varepsilon$.

Knowing that there exists techniques to monitor BLTL properties [13], the model checking of A-BLTL is rather evident. Given an A-BLTL property $\Phi_{1} \stackrel{\Omega}{\Rightarrow} \leq k$ $\Phi_{2}$, the monitor will first check whether the run satisfies $\Phi_{1}$ using classical runtime verification techniques. If no, then the property is not satisfied. If yes, then one checks whether there is a pair of two successive states between $t_{0}$ and $t_{0}+k$ that satisfies $\Omega$. The latter is done by parsing the run. If this pair does not exist, then the property is not satisfied. Else we start a new monitor from the suffix of the run starting in the second state of the pair in order to verify $\Phi_{2}$.

\subsection{Verifying unbounded SAS Properties using SMC}

We propose a method inspired by [21] to check unbounded A-BLTL properties. The principle is to combine a reachability analysis by model checking the underlying finite-state machine, with a statistical analysis of the stochastic model using the algorithms introduced previously.

We consider an A-BLTL property $\Phi \stackrel{\Omega}{\Rightarrow} \Psi$, where $\Phi$ and $\Psi$ are BLTL formulas. We first consider the reachability problem with objective $G=\left\{s \mid \exists s^{\prime} . s \rightsquigarrow\right.$ $\left.s^{\prime} \wedge \Omega\left(s, s^{\prime}\right)\right\}$. The preliminary to the statistical analysis is to compute the set $\operatorname{Sat}(\operatorname{Reach}(G))$, that is all the states of the SAS that may eventually reach a state in $G$. This can be computed using classical model checking algorithms for finite-state machines. Only the underlying automata of the DTMCs or CTMCs of the SAS is used for this analysis. As stochastic quantities are ignored, efficient symbolic techniques exist to speed up this process [6]. 


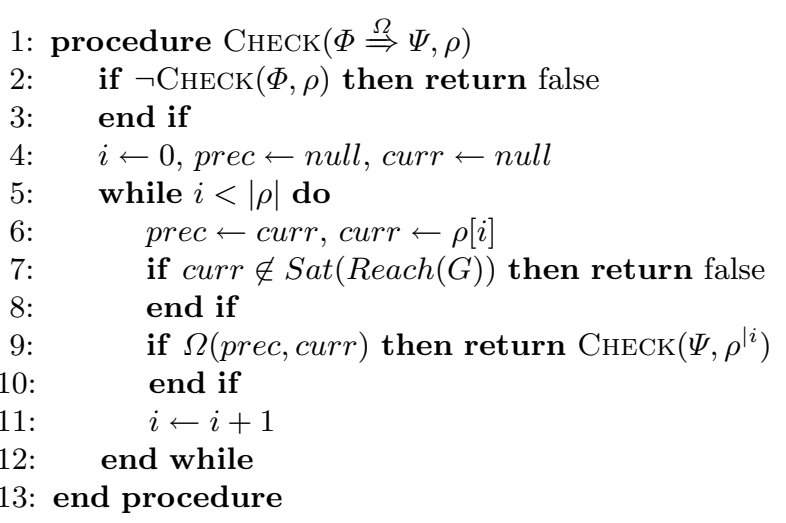

Fig. 2. Algorithm to monitor unbounded A-BLTL properties

Once this preliminary computation is performed, the CHECK algorithm from Figure 2 is used to monitor the runs of the SAS. The algorithm takes as input the A-BLTL property and a run $\rho$. The run should be in general infinite as there is no bound on the length of the runs that satisfied an unbounded A-BLTL property. In that case the states would be generated on-the-fly. The algorithm returns true or false, whether the run satisfied the property. We also denote $\operatorname{Check}(\Phi, \rho)$ as the monitoring of the BLTL property $\Phi$. Then the first step on line 2 is to monitor $\Phi$ on the run $\rho$. If the result is false then the property $\Phi \stackrel{\Omega}{\Rightarrow} \Psi$ is not satisfied. Otherwise the algorithm searches through $\rho$ for two states prec and curr such that $\Omega$ (prec,curr) is true. This is possible if curr belongs to the precomputed set $\operatorname{Sat}(\operatorname{Reach}(G))$. If $\Omega$ is satisfied the last step on line 10 is to monitor $\Psi$ from the current position in $\rho$.

For homogeneous Markov chains (with constant probability matrices, as it is the assumption in this paper), the algorithm almost surely (with probability 1) terminates, since it either reaches a state where $\Omega$ is unreachable, or the probability to reach two states that satisfy $\Omega$ is not null. It can be iterated to check sequences of adaptive operators, that is to say properties where $\Psi$ is also an unbounded A-BLTL.

\section{A software engineering point of view}

In this section, we propose high level formalisms to specify both adaptive systems and their properties. Then, we define semantics of those formalisms by exploiting the definitions introduced in the previous sections. This gives us a free verification technology for them. The situation is illustrated in Figure 3.

\subsection{Adaptive RML systems as a high level formalism for SAS}

We represent adaptive systems with Adaptive Reactive Module Language (ARML), an extension of the Reactive Module Languages (RML) used by the 


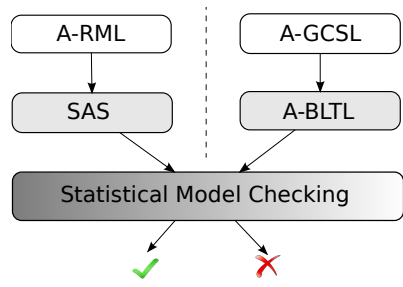

Fig. 3. SAS verification flow

PRISM toolset [16]. Due to space limit, the syntax common to RML and A-RML is only briefly described here ${ }^{1}$.

The RML language is based on the synchronisation of a set of modules defined by the user. A module is declared as a DTMC or CTMC, i.e., some local variables with a set of guarded commands. Each command has a set of actions of the form $\lambda_{i}: \mathrm{a}_{i}$ where $\lambda_{i}$ is the probability (or the rate) to execute $\mathrm{a}_{i}$. A-RML extends RML such that each module can have some parameters in order to define its initial state.

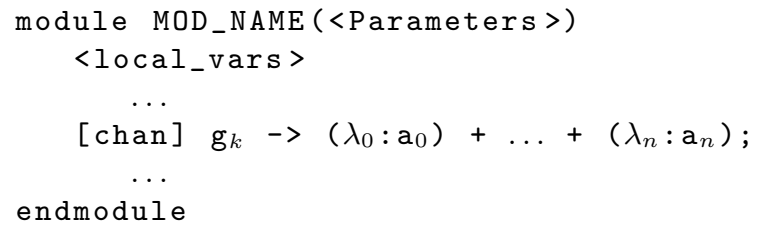

The optional channel identifiers prefixing commands are used to strongly synchronise the different modules of a RML system. A module is synchronised over the channel chan if it has some commands prefixed by chan. We say a command is independent if it has no channel identifier.

In A-RML a system is a set of modules and global variables. The modules synchronise on common channels such that the system commands are the independent commands of each module and the synchronised commands. A command synchronised on chan forces all the modules that synchronised over chan to simultaneously execute one of their enabled commands prefixed by chan. If one module is not ready, i.e. it has no enabled commands for chan, the system has no enabled command over chan. Similarly to a module, if the system reaches a state with a non-deterministic choice, it is solved by a stochastic behaviour based on a uniform distribution. This solution allows to execute the A-RML system in accordance with the DTMC/CTMC models.

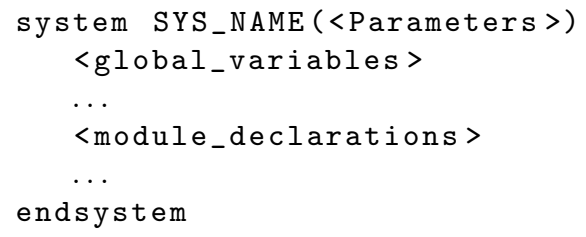

1 The full syntax can be found at http://project.inria.fr/plasma-lab/ 
An adaptive system consists in a set of different views, each represented by an A-RML system, and a list of adaptations represented by adaptive commands. The adaptive environment is used to specify which one is the initial view and what adaptations are possible.

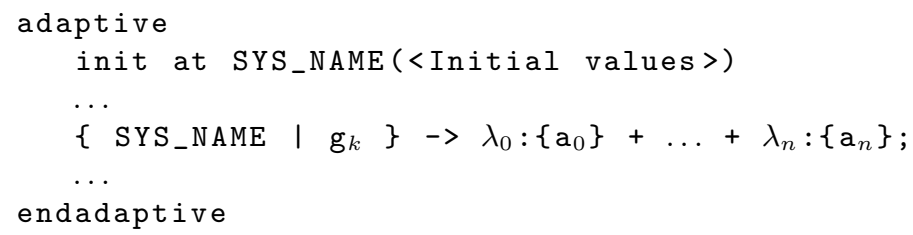

An adaptive command is similar to module command. It has a guard $\mathrm{g}_{k}$ that applies to the current view SYS_NAME, and a set of actions $\lambda_{i}: a_{i}$ where $\lambda_{i}$ is the probability (or the rate) to execute action $a_{i}=\operatorname{SYS}$ NAME' $^{\prime}\left(\mathrm{e}_{0}, \ldots, \mathrm{e}_{m}\right)$. This action defines the next view SYS_NAME' after performing the adaptation. This view is determined according to the states of the previous view by setting the parameters of SYS_NAME' with the expressions $\mathbf{e}_{0}, \ldots, \mathbf{e}_{m}$ evaluated over SYS NAME. The execution of the adaptive command is done in accordance with the SAS semantics.

Theorem 1. The semantics of A-RML can be defined in terms of SAS.

The proof of the above theorem is a direct consequence of the fact that semantics of RML is definable in terms of composition of MC, and that the definition of an adaptive command can also be represented as a MC.

\subsection{A contract language for SAS specification}

The Goal and Contract Specification Language (GCSL) was first proposed in [2] to formalise properties of adaptive systems in the scope of the DANSE project. It has a strong semantics based on BLTL but it has a syntax close to the hand written English requirements. Dealing with formal temporal logic is often an issue to formalise correctly the initial English requirements. Most of the time the formalisation frequently contains some mistakes, which is due to the nesting of the temporal operators. The difficulty for correctly specifying properties is enough to make the overall methodology useless.

The GCSL syntax combines a subset of the Object Constraint Language (OCL) [18] (used to define state properties, i.e., Boolean relations between the system components) and English behavioural patterns used to express the evolution of these state properties during the execution of the system. The usage of OCL is illustrated in Example 1.

Example 1. We consider a SAS describing the implementation of an emergency system in a city. The city area is divided as a set of districts where each district may have some equipment to fight against the fire, e.g. some fire stations with fire brigades and fire fighting cars. Each district is also characterised by a risk of fire and the considered damages are mainly related to the population size 
of each district. The requirement "Any district cannot have more than 1 fire station, except if all districts have at least 1" ensures the minimal condition for the equipment distribution in the city. We use syntactic coloration to make the difference between the parts of the language used in the property: the words in red are identifiers from the model, the blue part is from OCL, like collection handling, and the black words are variables:

City.itsDistricts $\rightarrow$ exists(district $\mid$ district.ownedFireStations $>1$ ) implies

City.itsDistricts $\rightarrow$ for All(district | district.ownedFireStations $\geq 1$ )

GCSL patterns are used to specify temporal properties. In this section we only present a subset of such patterns that is considered to be general enough to specify properties of a large set of industry-examples from the DANSE project. After having read this section, the user shall understand that the set can be easily increased. Each pattern can nest one or more state properties, denoted in the grammar by the non-terminals <OCL-prop> and <arith-rel>, that respectively denote a state property written in OCL or an arithmetic relation between the identifiers used in the model. The non-terminal <int > denotes a finite time interval over which the temporal pattern is applied, and $\langle\mathrm{N}\rangle$ is a natural number. The patterns can be used directly or combined with OCL: applying a pattern to a collection of system components defines a behavioural property that is applied to each element of the collection. We present below an excerpt of the complete GCSL grammar available in [2]:

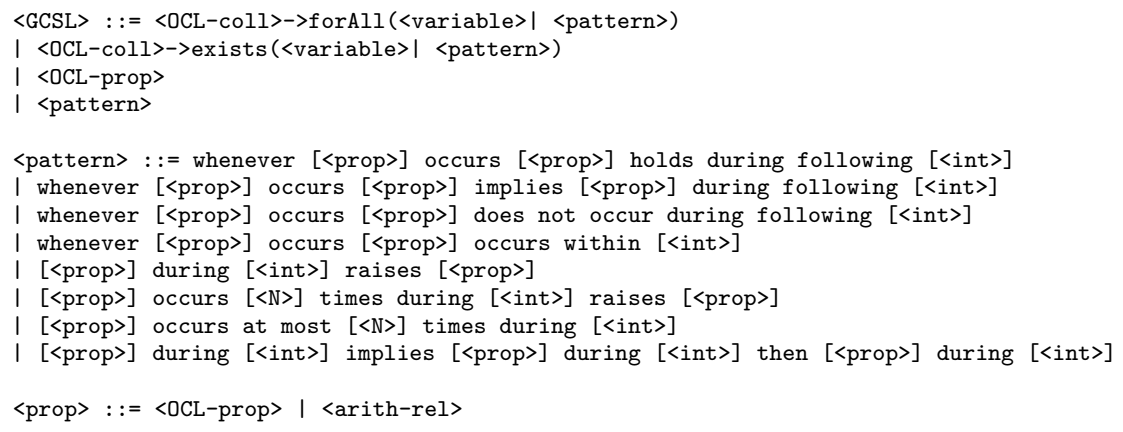

Example 2. Consider the following requirement about the model described in Example 1: "The fire fighting cars hosted by a fire station shall be used all simultaneously at least once in 6 months". This requirement uses both GCSL and OCL patterns:

City.itsFireStations $\rightarrow$ forAll(fStation | Whenever [fStation.hostedFireFightingCars $\rightarrow$ exists(ffCar | ffCar.isAtFireStation)] occurs, [fStation.hostedFireFightingCars $\rightarrow$ forall(ffCar $\mid$ ffCar.isAtFireStation $=$ false)] occurs within [6 months])

We now propose A-GCSL, a syntax extension for GCSL that can be used to describe adaptive requirements of SAS. A-GCSL extends the GCSL grammar by adding a new pattern that allows to express adaptive relations as done with the two adaptive operators defined in Section 3. The first pattern of <dyna-spec> is equivalent to the operator $\stackrel{\Omega}{\Rightarrow}$ and the second one denotes $\stackrel{\Omega}{\longrightarrow}$. Any adaptive 
requirement has three elements $(A, \Omega, G)$ that are called assumption, trigger and guarantee, respectively. The assumption and guarantee are specified in GCSL, whereas the trigger is in OCL. The syntax allows to compose the patterns by specifying the guarantee with an adaptive pattern. For instance, a composed requirement of the form if $\Phi_{1}$ holds and for all rule that satisfies $\Omega$ then (if $\Phi_{2}$ holds and for all rule that satisfies $\Omega^{\prime}$ then $\Phi_{3}$ holds) holds is equivalent to the property $\Phi_{1} \stackrel{\Omega}{\Rightarrow} \Phi_{2} \stackrel{\Omega^{\prime}}{\Longrightarrow} \Phi_{3}$. The A-GCSL grammar is the following:

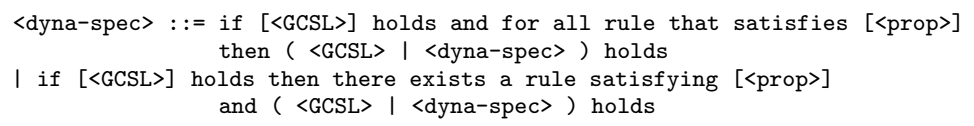

Example 3. Consider again the system in Example 1 and the following A-GCSL requirement:

if [ City.underFire $=0$ ] holds and for all rule such that rule satisfies [ $\mathrm{Ci}$ ty.underFire $\geq 3$ ] then [ City.itsDistricts $\rightarrow$ forall(district $\mid$ district.decl $=$ false $\Longrightarrow$ whenever [ district.decl $=$ true ] occurs, $[$ district.fire $=0$ ] occurs within [50 hours] ) ] holds

The attribute underFire denotes the number of districts in which a fire has been declared. If there are more than three fires in the city, then the fire stations change their usual emergency management into a crisis one. When such management is activated, the firemen have 50 hours to fix the problem. The requirement can be translated in A-GCSL using the following formula:

$$
\begin{aligned}
& \Phi_{6}=(\text { underFire }=0) \stackrel{\text { underFire } \geq 3}{\longrightarrow} \leq 10000 \\
& \bigwedge_{d_{i}: \text { district }}\left(\neg d_{i} \cdot \text { decl } \Longrightarrow G_{\leq 10000}\left(d_{i} \cdot \text { decl } \Longrightarrow F_{\leq 50} d_{i} . \text { fire }=0\right)\right)
\end{aligned}
$$

In [2], we have showed that any GCSL pattern can be translated into a BLTL formula. The result extends as follows.

Theorem 2. Any A-GCSL pattern can be translated into an A-BLTL property.

This result is an immediate consequence of the definition of the adaptive pattern.

\section{Experiments with SAS}

Our work has been implemented in a new statistical model checker named PlASMA-LAB [5], a platform that includes efficient SMC algorithms, a graphical user interface (GUI) and multiple plugins to support various modelling languages. The tool is written in Java that offers maximum cross-platform compatibility. The GUI allows to create projects and experiments, and it implements a simple and practical mean of distributing simulations using remote clients. Plasma-LAB also provides a library to write new plugins and create custom statistical model checkers based on arbitrary modelling languages. Developers will only need to 
implement a few simple methods to build a simulator and a logic checker, and then beneficiate from PLASMA-LAB SMC algorithms.

Plasma-LAB can be used as a standalone application or be instantiated within other softwares. It has already been incorporated in various performancecritical softwares and embedded hardware platforms. The current plugins allow to simulate biologic models, models written in RML and A-RML, but it has also the capabilities to drive an external engine to perform the simulations, like MATLAB, Scilab, or DESYRE a simulator for adaptive systems developed by Ales [1].

\subsection{CAE model}

Together with our industrial partners in the DANSE project, we have developed the Concept Alignment Example (CAE). The CAE is a fictive adaptive system example inspired by real-world Emergency Response data to a city fire. It has been built as a playground to demonstrate new methods and models for the analysis and visualization of adaptive systems designs.

The CAE describes the organization of the firefighting forces. We consider in our study that the city is initially divided into 4 districts, and that the population might increase by adding 2 more districts. Different and even more complex examples can be built using the components of this design.

A fire station is assigned to the districts, but as the fire might spread within the districts, the system can adapt itself by hiring more firemen. We can therefore design a SAS with three views as described in Figure 4.

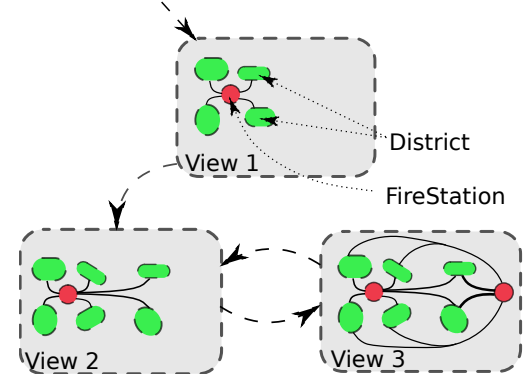

Fig. 4. Components and Views in the CAE model

Adaptive transitions exist between these views to reflect changes in the environment and adaptations of the system. Initially in View 1, the system can switch to View 2 when the population of the city increase. This change models an uncertainty of the environment, and for the purpose of this study we fix its probability to 0.01 Then, if the number of fires becomes greater than 2, the system adapts itself by switching to View 3 . If the number of fires eventually reduces and becomes lower than 2, the system might return to View 2. Again, as this change is uncertain, we fix its probability to 0.8 . 
We design several A-RML models of the system that consist in two types of modules: District and FireStation, both based on a CTMC semantics. First, we study a model AbstractCAE that is an abstract view of the SAS. In this model, the District module, presented below, is characterized by a constant parameter $\mathrm{p}$, that determines the probability of fire, and by two Boolean variables decl and men, that respectively defines if a fire has been declared and if the firemen are allocated to the district. The module fireStation has one constant parameter distance ${ }_{i}$ for each module of the system. This parameter determines the probability to react at a fire, such that the greater the distance, the lower the probability. However a fire station can only treat one fire at a time, which is encoded with a Boolean variable allocated. The fire stations and the districts synchronize on channels allocate and recover, that respectively allocate firefighters to the district and bring them back when the fire is treated. The different views are constructed by instantiating and renaming the modules presented above.

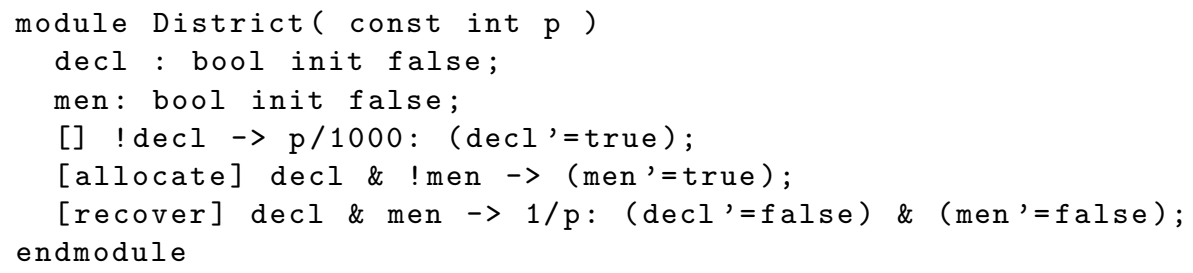

We refine this model to better encode the behaviour of the SAS. In this new model ConcreteCAE a new variable fire of module District ranges from 0 to 10 and grades the intensity of the fire. The fire stations can now assign several cars (from 0 to 5) to each districts. Therefore the variables men and allocated becomes integers.

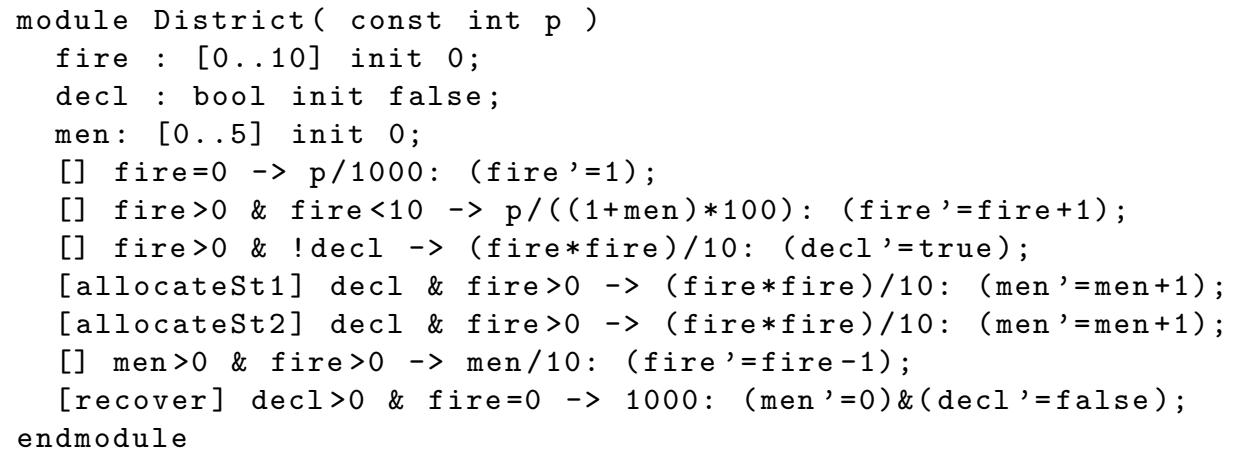

From the two models we can consider several subparts composed by one or several views of the SAS. Adaptive commands are used to model the transitions between the different views.

- AbstractCAE_1 consists in View 1 and 2 from model AbstractCAE.

- AbstractCAE_2 consists in View 2 and 3. 
- AbstractCAE_3 has the same views as AbstractCAE_2 but is initiated in View 3 instead of View 2.

- ConcreteCAE_1 only consists in View 1 from model ConcreteCAE.

- ConcreteCAE_2 only consists in View 2.

- ConcreteCAE_3 only consists in View 3.

- ConcreteCAE_Full is the full model of ConcreteCAE, with the 3 views and all the adaptive transitions between them.

\subsection{Checking requirements}

The requirements are expressed in A-GCSL and translated to A-BLTL. We first check the model AbstractCAE against A-BLTL properties with adaptive operators. Our goal is to verify that the transitions between the different views of the system occurs and satisfy some properties.

The first property, if [true] holds then there exists a rule satisfying [underfire $\leq 1]$ and Always [!maxfire], checks that when the system is in View 1, it eventually switches to View 2 when the number of districts that have declared a fire (underfire) is still lower than 1 , and that as a result the system remains safe for a limited time period, i.e., the number of districts that have declared a fire is not maximum (maxfire is false). To check this property we limit the analysis to the model AbstractCAE_1 with only View 1 and View 2. The A-GCSL property is translated in an A-BLTL formula: $\Phi_{1}=$ true $\stackrel{\text { underfire } \leq 1}{=} G_{\leq 1000}$ !maxfire, and the results in Table 2 show that the probability to satisfy the property is only $50 \%$. This justify the need to add a second fire station, as in View 3.

The second property, if [true] holds then there exists a rule satisfying [true] and Always [!maxfire], checks that from View 2 a second fire station is quickly added, which switches the system to View 3, and that then the system is safe. The property is checked on the model AbstractCAE_2 using the A-BLTL formula : $\Phi_{2}=$ true $\stackrel{\text { true }}{\Longrightarrow} \leq 100 G_{\leq 10000}$ !maxfire.

Finally, with the property if [true] holds then there exists a rule satisfying [true] and [true], we check that from View 3 the system eventually returns to View 2. Therefore we use the model AbstractCAE_3 that starts in View 3 and we check the A-BLTL formula $\Phi_{3}=$ true $\stackrel{\text { true }}{\Longrightarrow} \leq 100$ true.

The AbstractCAE models are simple enough to be able to perform reachability analyses and check the unbounded A-BLTL properties presented above using Algorithm 2. In a second step we consider the models ConcreteCAE to better evaluate the safety of the system. The state spaces of these models contain several millions of states, and therefore, they can only be analyzed by purely SMC algorithms. We verify the two following properties:

- Always !maxfire, to check that the maximum of fire intensity of 10 is never reached in any district. This corresponds to $\Phi_{4}=G_{\leq 10000}$ !maxfire.

- Whenever [fire >0] occurs [fire =0] within [50 hours], to check that a fire in a district is totally extinct within 50 hours. This corresponds to $\Phi_{5}=G_{\leq 10000}\left(\right.$ d6.fire $>0 \Longrightarrow F_{\leq 50}$ d6.fire $\left.=0\right)$. 
These two properties are first checked for each view of the system. The results in Table 2 show that while View 1 and View 3 are surely safe, View 2 is frequently unsafe. But when we check these properties on the complete adaptive model ConcreteCAE_Full, with the three views, we can show that the system remains sufficiently safe. It proves that after a change of the environment (the increase of population) the system is able to adapt itself to guaranty its safety.

In the last experiment of Table 2 we check the A-GCSL property presented in Example 3. This bounded adaptive A-GCSL property is checked using the full ConcreteCAE model.

We have performed each experiment in Plasma-LAB with a confidence $\delta=0.01$ and an error bound $\varepsilon=0.02$. The results in Table 2 give the probabilities estimation and the time needed to perform the computation.

\begin{tabular}{|c|c|c|c|}
\hline PROPERTY & CAE MODEL & ESTIMATION INTERVAL & Consumed Time \\
\hline$\Phi_{1}$ & $\begin{array}{l}\text { AbstractCAE_1 } \\
\text { View 1, View } 2\end{array}$ & {$[0.53,0.56]$} & $1351 \mathrm{~s}$ \\
\hline$\Phi_{2}$ & $\begin{array}{l}\text { AbstractCAE_2 } \\
\text { View 2, View } 3\end{array}$ & {$[0.84,0.86]$} & $11 \mathrm{~s}$ \\
\hline$\Phi_{3}$ & $\begin{array}{c}\text { AbstractCAE_3 } \\
\text { AbstractCAE_2 starting } \\
\text { from View } 3\end{array}$ & {$[0.98,1]$} & $1363 \mathrm{~s}$ \\
\hline$\Phi_{4}$ & $\begin{array}{c}\text { ConcreteCAE_6 } \\
4 \text { dist. } 1 \text { sta. }\end{array}$ & {$[0.95,0.99]$} & $\begin{array}{l}11 \mathrm{~s} \\
9 \mathrm{~s}\end{array}$ \\
\hline $\begin{array}{l}\Phi_{4} \\
\Phi_{5}\end{array}$ & $\begin{array}{c}\text { ConcreteCAE_2 } \\
6 \text { dist. } 1 \text { sta. }\end{array}$ & $\begin{array}{c}c[0.46,0.5] \\
{[0.21,0.25]}\end{array}$ & $\begin{array}{l}15 \mathrm{~s} \\
13 \mathrm{~s}\end{array}$ \\
\hline$\Phi_{4}$ & ConcreteCAE_3 & {$[0.98,1]$} & $30 \mathrm{~s}$ \\
\hline $\begin{array}{l}\Phi_{5} \\
\Phi_{4}\end{array}$ & $\frac{6 \text { dist. } 2 \text { sta. }}{\text { ConcreteCAE Full }}$ & $\frac{[0.98,1]}{[0.89,0.93]}$ & $\frac{31 \mathrm{~s}}{25 \mathrm{~s}}$ \\
\hline$\Phi_{5}$ & 4-6 dist. $1-2$ sta. & {$[0.82,0.86]$} & $42 \mathrm{~s}$ \\
\hline$\Phi_{6}$ & $\begin{array}{l}\text { ConcreteCAE_Full } \\
\text { 4-6 dist. } 1-2 \text { sta. }\end{array}$ & {$[0.47,0.51]$} & $109 \mathrm{~s}$ \\
\hline
\end{tabular}

Table 2. Experiments on CAE models

\section{Conclusion}

This paper presents a new methodology for the rigorous design of stochastic adaptive systems. Our model is general, but the verification procedure can only reason on a finite and known set of views. Our formalism is inspired from [24], where both the stochastic extension and high level formalisms are not considered. In future work, we will extend this approach to purely dynamic systems. Another objective is to extend the work to reason about more complex properties such as energy consumption. Finally, we shall exploit extensions of SMC algorithms such as CUSUM [19] which permits to reason on switches of probability satisfaction. This would allow us to detect emergent behaviors. 


\section{References}

1. Ales Corp.: Advanced laboratory on embedded systems, http://www .ales.eu.com/

2. Arnold, A., Boyer, B., Legay, A.: Contracts and behavioral patterns for systems of systems: The EU IP DANSE approach. In: AiSoS. EPTCS (2013)

3. Baier, C., Katoen, J.P.: Principles of Model Checking. The MIT Press (2008)

4. Basu, A., Bensalem, S., Bozga, M., Delahaye, B., Legay, A.: Statistical abstraction and model-checking of large heterogeneous systems. Int. J. Softw. Tools Technol. Transf. 14(1), 53-72 (Feb 2012)

5. Boyer, B., Corre, K., Legay, A., Sedwards, S.: Plasma-lab: A flexible, distributable statistical model checking library. In: QEST. LNCS, vol. 8054, pp. 160-164 (2013)

6. Burch, J.R., Clarke, E., McMillan, K.L., Dill, D., Hwang, L.J.: Symbolic model checking: 1020 states and beyond. In: LICS. pp. 428-439 (1990)

7. Cheng et al.: Software engineering for self-adaptive systems: A research roadmap. In: Software Engineering for Self-Adaptive Systems. LNCS, vol. 5525 (2009)

8. Clarke, E., Donzé, A., Legay, A.: On simulation-based probabilistic model checking of mixed-analog circuits. Form. Methods Syst. Des. 36(2), 97-113 (Jun 2010)

9. Clarke, Jr., E.M., Grumberg, O., Peled, D.A.: Model checking. MIT Press, Cambridge, MA, USA (1999)

10. Clarke, E., Faeder, J., Langmead, C., Harris, L., Jha, S., Legay, A.: Statistical model checking in biolab: Applications to the automated analysis of t-cell receptor signaling pathway. In: CMSB, LNCS, vol. 5307, pp. 231-250 (2008)

11. DANSE: Designing for adaptability and evolution in sos engineering (dec 2013), https://www.danse-ip.eu/home/

12. Havelund, K., Rosu, G.: Preface: Volume 70, issue 4. ENTCS pp. $201-202$ (2002), Runtime Verification

13. Havelund, K., Roşu, G.: Synthesizing monitors for safety properties. In: TACAS, LNCS, vol. 2280, pp. 342-356 (2002)

14. Hoeffding, W.: Probability inequalities for sums of bounded random variables. Journal American Statistical Association 58(301), 13-30 (March 1963)

15. Jha, S.K., Clarke, E.M., Langmead, C.J., Legay, A., Platzer, A., Zuliani, P.: A bayesian approach to model checking biological systems. In: CMSB. pp. 218-234 (2009)

16. Kwiatkowska, M., Norman, G., Parker, D.: PRISM 4.0: Verification of probabilistic real-time systems. In: CAV'11. LNCS, vol. 6806, pp. 585-591. Springer (2011)

17. Meyer, B.: Applying "design by contract". Computer 25(10), 40-51 (1992)

18. OMG: Ocl v2.2 (feb 2010), http://www.omg.org/spec/OCL/2.2/

19. Page, E. S.: Continuous inspection schemes. Biometrika 41(1/2), pp. 100-115 (1954)

20. Sen, K., Viswanathan, M., Agha, G.: On statistical model checking of stochastic systems. In: CAV. pp. 266-280 (2005)

21. Younes, H., Clarke, E., Zuliani, P.: Statistical verification of probabilistic properties with unbounded until. In: Formal Methods: Foundations and Applications. LNCS, vol. 6527, pp. 144-160. Springer (2011)

22. Younes, S., Clarke, E.M., Gordon, G.J., Schneider, J.G.: Verification and planning for stochastic processes with asynchronous events. Tech. rep. (2005)

23. Zhang, J., Cheng, B.H.C.: Model-based development of dynamically adaptive software. In: ICSE. ACM (2006)

24. Zhang, J., Cheng, B.H.: Using temporal logic to specify adaptive program semantics. Journal of Systems and Software 79(10), 1361 - 1369 (2006) 